\title{
STABLE OSCILLATION OF MEMS RESONATORS BEYOND THE CRITICAL BIFURCATION POINT \\ H.K. Lee ${ }^{l^{*}}$, J.C. Salvia ${ }^{2 \dagger}$, S. Yoneoka ${ }^{l}$, G. Bahl', Y.Q. Qu ${ }^{l}$, R. Melamud ${ }^{1+}$, S. Chandorkar ${ }^{l}$, M.A. Hopcroft ${ }^{1 *}$, B. Kim ${ }^{l \cdot}$, and T.W. Kenny ${ }^{I}$ \\ ${ }^{1}$ Department of Mechanical Engineering, Stanford University, Stanford, California, USA \\ ${ }^{2}$ Department of Electrical Engineering, Stanford University, Stanford, California, USA
}

\begin{abstract}
MEMS resonators have many useful features, but they can suffer performance limitations because of the limited linear range of motion of their micromechanical elements. Operation beyond the critical bifurcation limit is believed to cause significant instabilities and is generally discouraged [1]. However, the stability criterion for closed-loop oscillators differs from that for open-loop observation [2], thereby enabling operation of stable oscillators beyond the limit dictated by critical bifurcation. This paper describes the use of phase-controlled oscillators to prove stable operation beyond the critical bifurcation limit, which can directly improve the power handling capabilities of many micromechanical oscillators.
\end{abstract}

\section{INTRODUCTION}

MEMS resonators are considered as the next generation frequency reference because of their miniaturization-friendly characteristics [3]. Frequency references are an essential component of electronic devices, and quartz crystal-based references (quartz resonators) have dominated the market. Although quartz resonators have many advantages, they have become a bottleneck in the miniaturization of electronic devices because the process of manufacturing them is hard to integrate with the current semiconductor fabrication processes. However, MEMS resonators can be batch fabricated with a standard semiconductor fabrication process. Therefore, they have the potential to provide micro-scale frequency references at a low cost. Hence, they tend to be more adequate for frequency references in miniaturized electronics.

The power handling performance of MEMS resonator-based oscillators is of great interest since phase noise, which is one of the important characteristics of a frequency reference, often scales inversely with carrier power. Even though MEMS resonators offer many promising attributes, the phase noise performance of MEMS resonator-based oscillators needs to improve for use in radio communications. Since the phase noise, especially the far-from-carrier phase noise, decreases with larger carrier power, researchers have developed various methods to enhance the power handling capacities of MEMS resonator-based oscillators [4,5]. Agarwal et al. utilized the balance between the electrical softening effect and the mechanical stiffening effect to attain larger power handling in linear resonators [4].

Although larger vibration amplitude would be favorable for power handling performance, nonlinearities arise in most MEMS resonators, and the onset of critical bifurcation is believed to set the limit [1]. Since the output current from a MEMS resonator is proportional to the mechanical vibration amplitude, we can achieve larger power handling by increasing the range of motion of the micromechanical elements. However, the maximum range of motion has not been exploited because of the belief of the instability at large amplitude [1]. Most MEMS resonators have nonlinearities that arise when the vibration amplitude is large, and they cause the amplitude dependent frequency change, also called the Duffing or A-f effect, and this phenomenon is well understood $[2,6]$. If the amplitude becomes larger than the critical bifurcation point, multiple amplitude solutions exist for some driving frequencies, as shown in Figure 1, where resonators can lose stability. Open-loop observation of the amplitude-frequency (A-f) curve of resonators using a network analyzer reinforced this idea. When a resonator operates in the nonlinear regime, part of the A-f curve is unobservable in open-loop measurement because of instability in the vicinity of the critical bifurcation point. In addition, designers often associate the idea of instability in open-loop observation with instability in closed-loop system [1]. Thus, the critical bifurcation point has constrained the maximum vibration amplitude of MEMS resonator-based oscillators.

The stability criteria for the open-loop system cannot be directly applied to the closed-loop system since they have different driving conditions. While a network analyzer sets a driving frequency during an open-loop measurement, the feedback circuit in a closed-loop oscillator sets a phase between the input and output of a resonator. Because of this difference, the stability condition of a closed-loop system differs from that for an open-loop system; a resonator within a closed-loop system is stable in any amplitude and frequency condition, regardless of the critical bifurcation [2]. In order to experimentally demonstrate stable operation of MEMS resonator-based oscillators beyond the critical bifurcation point, we utilized a phase-controlled oscillator to measure A-f points that constitute the A-f curve.

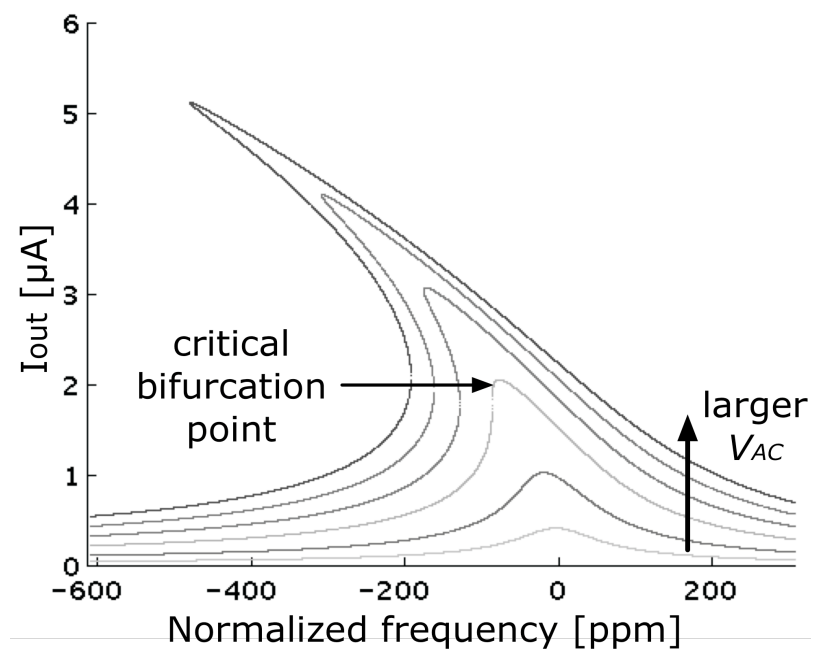

Figure 1: Theoretical amplitude-frequency (A-f) curves of a resonator at various driving conditions. When an A-f curve is below the critical bifurcation point, the frequency-to-amplitude relation is a one-to-one function. However, when an A-f curve is above the critical bifurcation point, the frequency-to-amplitude relation becomes one-to-many for some frequencies. 


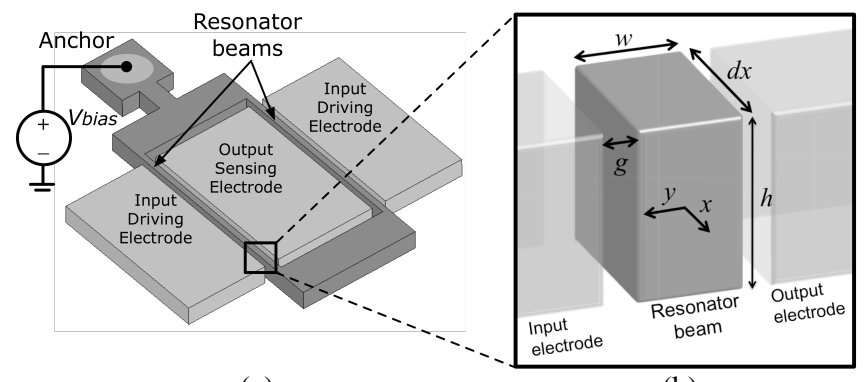

(a)

(b)

Figure 2: (a) Single-anchored double-ended tuning fork resonator for this study. (b) Differential element of a resonator beam. $x$ is the longitudinal direction, and $y$ is the direction of beam displacement. $h$ is the height of the beam, $w$ is the width of the beam, and $g$ is the size of the transduction gap.

\section{THEORY}

\section{Flexural Mode Beam Resonator}

We used single-anchored double-ended tuning fork (SA-DETF) type flexural mode silicon resonators for this study. We have fabricated MEMS resonators by using the 'epi-seal' process introduced in [7]. Among many types of resonators that can be realized under the 'epi-seal' process, DETF type resonators have shown reliable performance [8]. In addition, single-anchored resonators experience only the electrical softening effect in the first order [5]; thus, they go into the nonlinear regime easily and predictably. Therefore, SA-DETF resonators are appropriate for this study. Figure $2 \mathrm{a}$ shows the SA-DETF resonator that we used for the experiment. The beams are $200 \mu \mathrm{m}$ long, $6 \mu \mathrm{m}$ wide, and $20 \mu \mathrm{m}$ thick. The transduction gap is $1.5 \mu \mathrm{m}$. The resonant frequency of the resonator is about $1.33 \mathrm{MHz}$.

We operate resonators by using electrostatic actuation and sensing, and the nonlinearity arises from the electrostatics. For actuation, we apply the bias voltage $\left(V_{\text {bias }}\right)$ to the resonant beams, and supply a stimulus $\mathrm{AC}$ signal $\left(v_{A C}\right)$ to the input electrodes. In order to detect the output signal by using capacitive sensing, we virtually ground the output electrode. Then, the net electrostatic force on a differential element (Fig. 2b) is given as follows:

$$
f_{E S}(x, t)=\frac{\varepsilon_{0} h}{2}\left[\frac{\left(V_{\text {bias }}-v_{A C}(t)\right)^{2}}{(g-v(x, t))^{2}}-\frac{V_{\text {bias }}^{2}}{(g+v(x, t))^{2}}\right]
$$

where $\varepsilon_{0}$ is the vacuum permittivity, $v$ is the beam displacement in $\mathrm{y}$-direction, and $f_{E S}$ is position and time dependent electrostatic force for unit length of the beam. If we combine equation 1 with the force balance equation of the differential element, we get

$$
\rho_{\text {mass }} \frac{\partial^{2} v}{\partial t^{2}}+c \frac{\partial v}{\partial t}=-B \frac{\partial^{4} v}{\partial x^{4}}+f_{E S}(x, t)
$$

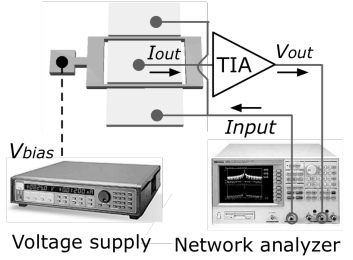

(a)

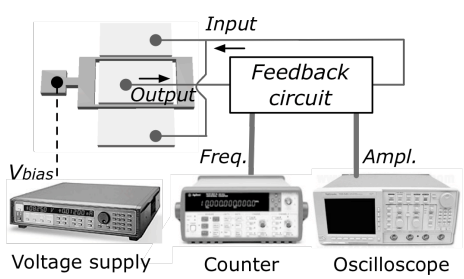

(b)
Figure 3: (a) Network analyzer setup for an open-loop observation. Voltage supply sets bias voltage $\left(V_{\text {bias }}\right)$, and trans-impedance amplifier (TIA) converts output current to voltage. (b) Oscillator setup for a closed-loop observation.

where $\rho_{\text {mass }}$ is the mass per unit length, $c$ is the damping coefficient, and $B$ is the bending stiffness. Since the electrostatic force term in equation 2 is nonlinear with respect to $v$, as shown in equation 1, the resonator system described in equation 2 is a nonlinear system.

In order to estimate the nonlinear behavior of a resonator, we use an approximate method. First, we approximate the electrostatic force term in equation 2 by using the Taylor series expansion with the assumption of small $v$.

$f_{E S}(x, t) \simeq \frac{\varepsilon_{0} h V_{\text {bias }}}{g^{2}}\left[v_{A C}(t)+\frac{2 V_{\text {bias }}}{g} v(x, t)+\frac{4 V_{\text {bias }}}{g^{3}} v(x, t)^{3}\right]$

After that, we can estimate the vibration amplitude and the resonant frequency at each of driving amplitudes by using the harmonic balance method [6].

\section{Stability Criterion for Each Driving Condition}

An open-loop system and a closed-loop system have totally different driving conditions. Figure 3 a shows a resonator within the open-loop system. A network analyzer in the open-loop system provides $v_{A C}$ with a given frequency and measures the response at that frequency, and it obtains the A-f curve of a resonator by measuring at many frequency values. In short, the network analyzer sets a driving frequency. A resonator within a closed-loop system appears in Figure 3b. Unlike the network analyzer in the open-loop system, a feedback circuit in the closed-loop system determines a phase between the output current, or equivalently $v$, and $v_{A C}$. Although the circuit usually sets $v_{A C}$ to lag $v$ by $90^{\circ}$ for the maximum vibration amplitude, other values of phase still can be used.

The stability conditions for both systems differ since they have different driving conditions, and stability is always guaranteed in a closed-loop system. With regard to the stability criterion for an open-loop system and a closed-loop system, Yurke et al. demonstrated rigorous mathematical derivation [2]. Besides,

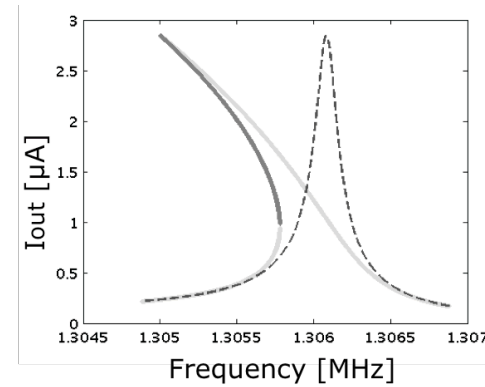

(a)

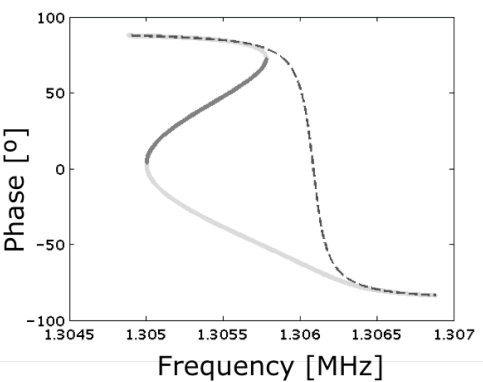

(b)

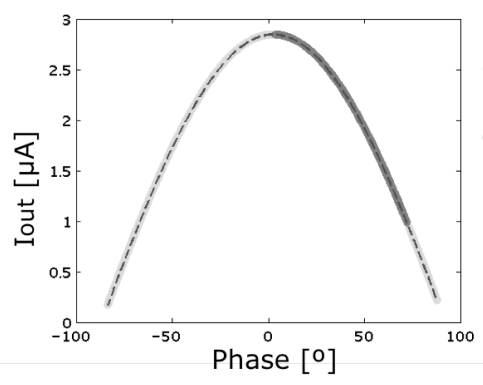

(c)

Figure 4: Theoretical amplitude-frequency-phase relation when a resonator is in the nonlinear regime (solid lines). The same relation when nonlinearities are not considered is also drawn for comparison (dashed lines). Entire solid lines are stable, thus observable, from closed-loop observation with a phase-controlled oscillator. However, parts of solid lines are unstable from open-loop observation. 
we can develop intuition for the result of [2] from the frequency-amplitude-phase relation of a resonator in the nonlinear regime. Figure 4 contrasts the nominal frequency-amplitude-phase relation of a resonator that is in the nonlinear and the linear regimes. In the nonlinear case, the frequency-to-amplitude relation, shown in Figure $4 \mathrm{a}$, is one-to-many for some frequencies. Therefore, the amplitude of a resonator is indeterminate for those driving frequencies, and this phenomenon causes instability in the open-loop system that sets a driving frequency. In addition, part of the A-f curve of a resonator in the nonlinear regime is unobservable because of the instability. However, the phase-to-amplitude and the phase-to-frequency relations are always a one-to-one function regardless of the nonlinearity, as shown in Figure 4b-c. For any given phase, a unique amplitude-frequency solution exists. Therefore, instability does not occur in the closed-loop system that sets an input-to-output phase rather than a driving frequency. Thus, the entire A-f curve becomes observable and stable oscillation above the critical bifurcation point becomes possible in a closed-loop oscillator.

\section{THE EXPERIMENTAL SETUP}

We can measure a resonator's nonlinear A-f curve from a closed-loop system simply by implementing the phase-controlled feedback circuit. From the preceding section, we know that stable oscillation is possible at any driving condition when a resonator is in a closed-loop system. However, the closed-loop oscillator with a fixed input-to-output phase can give us only one frequency and amplitude pair that corresponds to a phase. In other words, we can measure one A-f point in the entire A-f curve by utilizing the fixed-phase closed-loop system. Since we need to measure the entire A-f curve that comprises multiple A-f points, we need to be able to access multiple A-f points using a closed-loop system. Therefore, a closed-loop system with controllable phase is necessary for this study.

We built an experimental setup with a minor modification to a standard feedback circuit to incorporate a phase shifter for phase controllability, as shown in Figure 5. The phase shifter stage allows manual control of the feedback circuit's phase in two stages in order to provide wide phase range. Automatic level control including a variable gain amplifier maintains the input driving voltage, $v_{A C}$. After the modification, the feedback circuit was integrated to the closed-loop system shown in Figure $3 \mathrm{~b}$. An oscilloscope monitors the output of the trans-impedance amplifier to estimate the output current amplitude, and a frequency counter measures the frequency of oscillation. Then, we used the experimental setup to measure the A-f points that constitute the A-f curve, including operation points that are not accessible to ordinary fixed-phase oscillators.

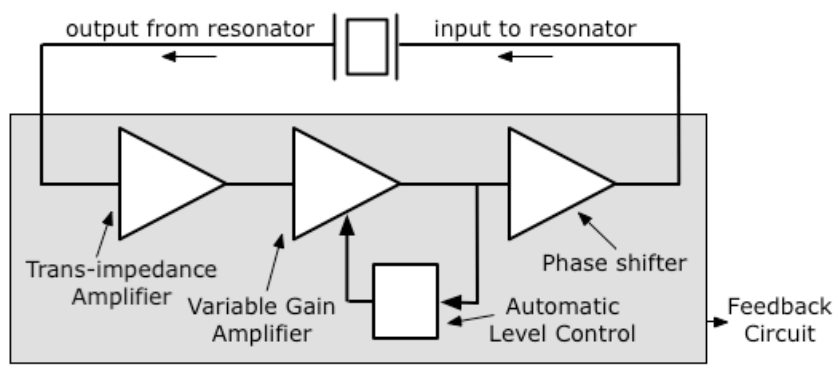

Figure 5: Circuit diagram of the phase-controlled feedback circuit. The trans-impedance amplifier (TIA) converts current to voltage. The variable gain amplifier and automatic level control maintain the drive amplitude $\left(v_{A C}\right)$, and the phase shifter sets the phase.

\section{RESULTS}

We measured the A-f characteristic of the resonator, shown in Figure $1 \mathrm{a}$, at several $V_{\text {bias }}$ and $v_{A C}$ conditions by using the phase-controlled closed-loop system. Since we wanted to measure the output current around the critical bifurcation as well as the maximum achievable output current in the system, we decided to use many $V_{\text {bias }}$ and $v_{A C}$ values: $45 \mathrm{~V}$ and $60 \mathrm{~V}$ for $V_{\text {bias }}$, and $0.18 \mathrm{~V}$, $0.30 \mathrm{~V}$, and $0.42 \mathrm{~V}$ for $v_{A C}$ amplitude. We manually changed the input-to-output phase of the feedback circuit shown in Figure $3 b$, and measured amplitude and frequency values at various phase values. The measured nonlinear A-f curves appear in Figure 6.

We demonstrated stable oscillation of the resonator above the critical bifurcation point, and improved the power handling by exploiting the nonlinear regime. We could obtain nonlinear A-f curves, which appear in Figure 6, from the closed-loop system. Thus, we experimentally proved the stability of a resonator in the nonlinear regime. In addition, Figure 7 shows a time-domain waveform at the highest current amplitude. Since the waveform is stable, we can further state the stability above the critical

(a) Measured A-f curve (Vbias $=45 \mathrm{~V}$ )

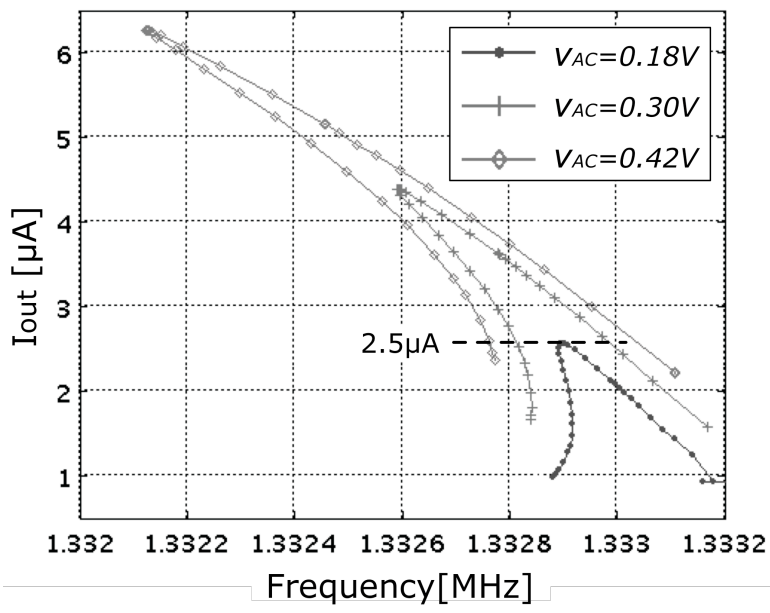

(b) Measured A-f curve (Vbias $=60 \mathrm{~V})$

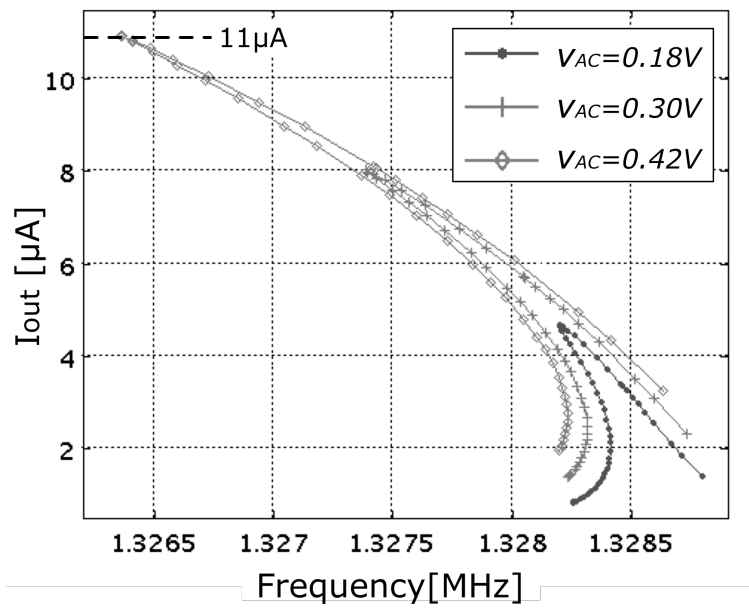

Figure 6: Measured A-f curves using the phase-controlled feedback circuit for 3 different values of $v_{A C}$ and 2 different values of $V_{\text {bias }}$. Numerous phase values are used for each driving condition to measure A-f points (points with a marker) that constitute an A-f curve. More nonlinear frequency shift is observed at larger $V_{\text {bias }}$ and $v_{A C}$ values. 


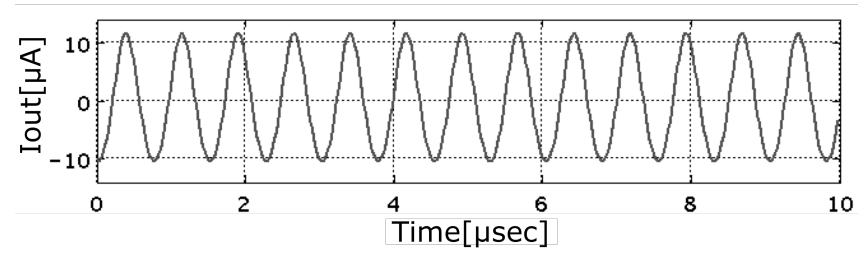

Figure 7: The output waveform of a resonator, which is converted from the output waveform of the trans-impedance amplifier of the feedback circuit (Fig.5), when the resonator is at the operating point with the highest current amplitude on Figure 6. Stable oscillation much above the critical bifurcation point is shown to be possible.

bifurcation point. In terms of the power handling improvement, we can compare the bottommost curve of Figure $6 \mathrm{a}$ and the uppermost curve of Figure $6 \mathrm{~b}$. The bottommost curve shows the A-f curve just above the critical bifurcation point and the uppermost curve shows the A-f curve with the largest power handling from this experiment. Comparing the peak current amplitude of two curves, we can notice that the peak of the uppermost curve is 4.4 times larger than that of the bottommost curve. In other words, the power handling performance improves by almost $20 x$ by exploiting the nonlinear regime.

\section{CONCLUSIONS}

In this study, we demonstrate stable oscillation of MEMS resonator-based oscillators beyond the critical bifurcation point. Considering that the maximum drive limit has not yet been determined, there is still further room for improvement of the current handling, thereby the power handling. In addition, the critical bifurcation point has constrained the resonator design optimization process [1,5]. Since it is no longer valid constraint, we now have more degrees of freedom in the design of MEMS resonators. Thus, we may design resonators with improved performance. Also, we can utilize the phase-controlled feedback circuit as a measurement tool to observe the nonlinear A-f characteristic of MEMS resonators. Since a part of nonlinear A-f curve is unobservable in open-loop observation with a network analyzer, the experimental setup in this study provides a better nonlinear characterization method. Therefore, it allows more capable analysis of nonlinear behaviors of MEMS resonators.

Future study will include the research on other limiting factors and on the phase noise performance. Although the critical bifurcation does not limit the vibration amplitude, it is highly probable that other mechanisms such as pull-in [9] limit the amplitude. Therefore, we will investigate other limiting mechanisms for the ultimate power handling performance. In addition, research on the phase noise performance in the nonlinear regime is ongoing since it is suspected that nonlinearities may worsen phase noise even if the power handling is improved [10].

\section{ACKNOWLEDGEMENTS}

This work was supported by DARPA HERMIT (ONR-N66001-03-1-8942), the Bosch Palo Alto Research and Technology Center, the Samsung Scholarship, the National Science Foundation Graduate Fellowship, and the National Users Network facilities funded by the National Science Foundation under award ECS-9731294. The authors would especially like to thank Clark Nguyen, Amit Lal, and Andrei Shkel for their DARPA HERMIT program management.

$\dagger$ currently at SiTime Corporation, Sunnyvale, CA, USA

\$ currently at Hewlett-Packard Laboratories, Palo Alto, CA, USA

- currently at Sandia National Laboratories, Albuquerque, NM, USA

\section{REFERENCES}

[1] V. Kaajakari, T. Mattila, A. Oja, and H. Seppä, "Nonlinear Limits for Single-Crystal Silicon Microresonators", Journal of Microelectromechanical Systems, 13, 5 (2004).

[2] B. Yurke, D.S. Greywall, A.N. Pargellis, and P.A. Busch, "Theory of Amplifier-Noise Evasion in an Oscillator Employing a Nonlinear Resonator", Physical Review A, 51, 5 (1995).

[3] C.T.-C. Nguyen, "MEMS Technology for Timing and Frequency Control", IEEE Transactions on Ultrasonics, Ferroelectrics, and Frequency Control, 54, 2 (2007).

[4] M. Agarwal, S.A. Chandorkar, R.N. Candler, B. Kim, M.A. Hopcroft, R. Melamud, C.M. Jha, T.W. Kenny, and B. Murmann, "Optimal Drive Condition for Nonlinearity Reduction in Electrostatic Microresonators", Applied Physics Letters, 89, 214105 (2006).

[5] M. Agarwal, Nonlinearities and Phase Noise in Electrostatically-Transduced-MEMS-Resonator Based Oscillators, Ph.D. dissertation at Stanford University, California, 2007.

[6] C. Hayashi, Nonlinear Oscillations in Physical Systems, Princeton University Press, New Jersey, 1985.

[7] R.N. Candler, M.A. Hopcroft, B. Kim, W.-T. Park, R. Melamud, M. Agarwal, G. Yama, A. Partridge, M. Luts, and T.W. Kenny, "Long-Term and Accelerated Life Testing of a Novel Single-Wafer Vacuum Encapsulation for MEMS Resonators", Journal of Microelectromechanical Systems, 15, 6 (2006).

[8] B. Kim, R.N. Candler, M. Hopcroft, M. Agarwal, W.-T Park, and T.W. Kenny, "Frequency Stability of Encapsulated MEMS Resonator", Sensors and Actuators, A: Physical, 136, 125 (2007).

[9] A.H. Nayfeh, M.I. Younis, E.M. Abdel-Rahman, "Dynamic Pull-In Phenomenon in MEMS Resonators", Nonlinear Dynamics, 48, 153 (2007).

[10] V. Kaajakari, J.K. Koskinen, and T. Mattila, "Phase Noise in Capacitively Coupled Micromechanical Oscillators", IEEE Transactions on Ultrasonics, Ferroelectrics, and Frequency Control, 52, 12 (2005).

\section{CONTACT}

*H.K. Lee, tel: +1-650-796-9882; hyungkyu@stanford.edu 